\title{
Problems and future approaches for assessment of periodontal disease
}

\author{
Toshihiro Ansai *, Shuji Awano and Inho Soh \\ Division of Community Oral Health Development, Kyushu Dental University, Kitakyushu, Japan \\ ${ }^{*}$ Correspondence: ansai@kyu-dent.ac.jp \\ Edited by: \\ Alexandrina L. Dumitrescu, Dental Private Practice, Romania \\ Reviewed by: \\ Koji Inagaki, Aichi-Gakuin University Junior College, Japan
}

Keywords: periodontal disease, probing, bleeding from gingiva, questionnaire, epidemiology

Clinical diagnosis of periodontal disease is generally obtained by probing or attachment loss. Those have also been used in epidemiological investigations, though several problems have been pointed out, such as follows: infectious aggression by the dental instrument, time-consuming for both dental professionals and patients, and patient discomfort (e.g., pain or itching during probing).

For standardization of assessment of periodontal disease, calibration of probing between inter-examiners is needed. A systematic review of the periodontal literature has been conducted to assess the extent of reporting of calibration of probing. Searches of four electronic databases (Medline, Embase/BIDS, PubMed, and Cochrane Library) were undertaken. Search constraints were: 1996-2009; clinical trials; human studies; English language. Also, hand searches of 12 periodontal/dental journals were undertaken for 14-year period. Then, a total of 1037 papers from 668 citations as the electronic search and 369 citations as the hand search were reviewed. Of those papers, only $28 \%$ reported examiner alignment for that assessment, while $42 \%$ gave no information on examiner alignment and assessment (1). Furthermore, in 288 papers that reported the statistical methods for determining examiner alignment and assessment, approximately $20 \%$ noted kappa value, a standard index used to show whether an adequate agreement between examiners was guaranteed ( $>0.8$ better), while no description regarding such statistics were found in $66 \%$ of those articles (1).

Although it is important to begin by considering the definition of periodontal disease, no uniform case definition exists.
Gingival inflammation (gingivitis) (2), deepened pockets (3), periodontal bone loss (4), and a combination of different oral parameters (5) have been used to define periodontal disease. To further complicate the matter, a wide variation of cut-off values for these parameters have been utilized to identify the presence of periodontal disease, such as attachment loss greater than $3 \mathrm{~mm}$ per year (6), attachment loss greater than $3 \mathrm{~mm}$ and probing depth greater than $4 \mathrm{~mm}$ in at least $30 \%$ of sites measured (7), attachment loss greater than $4 \mathrm{~mm}$ in at least $30 \%$ of sites measured (8), and classification based on the proportion of teeth with greater than $4 \mathrm{~mm}$ of probing depth (9). Therefore, comparisons of results from different studies include uncertainty and it is difficult to make conclusions from metaanalyses. Thus, since the parameters used for diagnosis of periodontal disease are not straightforward, we consider that a probing method is inappropriate for determination of underlying disease status.

We would propose the following four methods as alternatives to probing for use in epidemiological studies and populationbased community examination: (1) number of lost teeth, (2) bleeding from gingiva, (3) self-reported questionnaire of periodontal condition, and (4) combination of general medical assessment and noninvasive tests of the gingiva. Problems with these methods and future perspective are discussed below.

(1) Number of lost teeth: tooth loss present an accumulative burden throughout life. As noted by Tu and Gilthorpe (10), tooth loss might be a better indicator than probing as a marker of lifetime oral health, and is less prone to measurement error. Importantly, a kappa value greater than 0.9 can be obtained for calibration of assessment of tooth loss, indicating good reliability. On the other hand, it is not clear whether tooth loss is the result of periodontal disease or dental caries or both. For example, according to a dental professional survey conducted in 2005 $(n=2001)$, the main reason for tooth loss in $42 \%$ of the subjects was periodontal disease, while dental caries was noted in $33 \%$, though gender and age differences were also found, with periodontal disease predominant in subjects over 45 years old (11). Another controversial issue is whether tooth loss itself reduces pathological condition related to periodontal disease. In other words, though tooth loss shows a past condition of inflammation, gingival inflammation related to periodontal condition may not be present.

(2) Bleeding from gingiva: the pathogenesis of periodontal disease includes existence of gingival inflammation, thus it is reasonable to assess bleeding from the gingiva. To date, gingival bleeding has been used for clinical diagnosis of periodontal disease. Recently, a test kit for simple assessment of occult blood has been developed in Japan, and found to be effective for screening of periodontal disease, with a sensitivity and specificity of 0.72 and 0.52 , respectively (9). Furthermore, multivariate logistic regression analysis performed in that study showed that the results of the test were significantly associated with the proportion of teeth 
with gingival bleeding and with probing depth greater than $4 \mathrm{~mm}$, even after adjusting for several confounding factors. The most important feature of the kit is its non-invasive design, since saliva is used as the test sample. Briefly, each subject refrained from eating and drinking from the previous night. For specimen collection, each rinsed their mouth for $10 \mathrm{~s}$ with $3 \mathrm{ml}$ of distilled water, then spat it into a small paper cup. We think that bleeding from the gingiva would be a better indicator of present periodontal tissue condition than tooth loss.

(3) Self-administrated questionnaire: recently, self-reported means for determining periodontal disease have received attention, primarily due to their simplicity and low cost. In an assessment of the validity of self-reported questionnaires, improvements in sensitivity and specificity occurred when such questions, as "Gum surgery in the past?", "Sore gums in the past?", "Scaling in the past?", "Bleeding gums now?", "Periodontal surgery in the past 2 years?", and "Chewing satisfaction?" were included (12). Furthermore, Eke et al. (13) reported that the combined effects of demographic measures (age, sex, race/ethnicity, education, smoking, etc.) and responses to five self-reported questions had a sensitivity of $85 \%$ and specificity of $58 \%$ for predicting periodontitis of mild or greater severity, and produced an area under the curve (ROC) of 0.81 .

(4) Combination of general medical assessment and non-invasive tests for gingiva: recent studies have shown bidirectional interrelationships between periodontal disease and systemic condition including metabolic syndrome, diabetes, and obesity (14-17). Recently, Japanese researchers investigated the association between gingival bleeding as a means of screening for periodontal disease and metabolic syndrome-related factors among 488 subjects aged 40-74 years who underwent specific health checkups. The subjects were divided into two groups, screen-positive and -negative. The results of logistic regression analyses showed that the adjusted odds ratios for obesity and metabolic syndrome in the screen-positive group were 1.64 (95\% confidence interval, 1.03-2.61) and 2.49 (1.34-4.63), respectively (18). Those authors concluded that there was a significant association between gingival bleeding in saliva and metabolic syndrome.

Based on those results showing an association between periodontal disease and metabolic syndrome, we propose a novel approach that utilizes a combination of general medical assessment including assessment for metabolic syndrome and simple assessment of gingival bleeding. Our concept is consistent with the common risk factor approach proposed by Sheiham and Watt (19). They noted that non-communicable disease is associated with common risk factors such as diet, smoking, stress, alcohol, physical fitness, and others, and suggested that an approach for oral health promotion should be preceded as a collaborative approach including such common risk factors, while conventional oral health approaches were neither effective nor efficient. Furthermore, in many developed countries including Japan, low participation in routine screening examinations annually performed for community-dwelling individuals remains an unsolved issue. As a result, our novel approach may provide a solution, since such medical examinations are supported by medical law of the Ministry of Health in Japan.

In this article, we examined several approaches used for periodontal assessment, including those lacking periodontal probing. We think that elimination of probing is an urgent issue in dental medicine. Furthermore, objective comparisons of nationwide and international data regarding periodontal disease would be indispensable for planning oral health strategies for general population. Therefore, we suggest construction of a novel consensus for international standardization regarding assessment of periodontal disease in the near future. In addition, standardized non-probing assessments must become standard.

\section{REFERENCES}

1. Hefti AF, Preshaw PM. Examiner alignment and assessment in clinical periodontal research.
Periodontol 2000 (2012) 59:41-60. doi:10.1111/j. 1600-0757.2011.00436.x

2. Buhlin K, Gustafsson A, Håkansson J, Klinge B. Self-reported oral health, dental care habits and cardiovascular disease in an adult Swedish population. Oral Health Prev Dent (2003) 1(4):291-9.

3. Hujoel PP, Drangsholt M, Spiekerman C, DeRouen TA. Periodontal disease and coronary heart disease risk. JAMA (2000) 284(11):1406-10. doi:10.1001/ jama.284.11.1406

4. Beck J, Garcia R, Heiss G, Vokonas PS, Offenbacher S. Periodontal disease and cardiovascular disease. J Periodontol (1996) 67(10 Suppl):1123-37. doi:10. 1902/jop.1996.67.10s.1123

5. Mattila KJ, Valtonen VV, Nieminen M, Huttunen JK. Dental infection and risk of new coronary events: perspective study of patients with documented coronary artery disease. Clin Infect Dis (1995) 20(3):588-92. doi:10.1093/clinids/20.3.588

6. Brown LF, Beck JD, Rozier RG. Incidence of attachment loss in community-dwelling older adults. $J$ Periodontol (1994) 65(4):316-23. doi:10.1902/jop. 1994.65.4.316

7. Armitage GC. Development of a classification system for periodontal diseases and conditions. Ann Periodontol (1999) 4(1):1-6. doi:10.1902/annals. 1999.4.1.1

8. Davila-Perez C, Amano A, Alpuche-Solis AG, Patiño-Marin N, Pontigo-Loyola AP, Hamada $\mathrm{S}$, et al. Distribution of genotypes of Porphyromonas gingivalis in type 2 diabetic patients with periodontitis in Mexico. J Clin Periodontol (2007) 34(1):25-30. doi:10.1111/j.1600-051X. 2006.01011.x

9. Shimazaki Y, Akifusa S, Takeshita T, Shibata Y, Doi Y, Hata J, et al. Effectiveness of the salivary occult blood test as a screening method for periodontal status. J Periodontol (2011) 82:581-7. doi:10.1902/ jop.2010.100304

10. Tu Y-K, Gilthorpe MS. Commentary: is tooth loss good or bad for general health? Int J Epidemiol (2005) 34:475-6. doi:10.1093/ije/dyi005

11. Aida J, Ando Y, Akhter R, Aoyama H, Masui M, Morita M. Reasons for permanent tooth extractions in Japan. J Epidemiol (2006) 16:214-9. doi: 10.2188/jea.16.214

12. Genco RJ, Falkner KL, Grossi S, Dunford R, Trevisan $M$. Validity of self-reported measures for surveillance of periodontal disease in two western New York population-based studies. J Periodontol (2007) 78(7 Suppl):1439-54. doi:10.1902/jop. 2007.060435

13. Eke PI, Dye BA, Wei L, Slade GD, ThorntonEvans GO, Beck JD, et al. Self-reported measures for surveillance of periodontitis. J Dent Res (2013) 92(11):1041-7. doi:10.1177/0022034513505621

14. Fentoglu O, Bozkurt FY. The bi-directional relationship between periodontal disease and hyperlipidemia. Eur J Dent (2008) 2:142-6.

15. Saito T, Shimazaki Y, Sakamoto M. Obesity and periodontitis. N Engl J Med (1998) 339:482-3. doi:10.1056/NEJM199808133390717

16. Tayler GW. Bidirectional interrelationships between diabetes and periodontal diseases: an epidemiologic perspective. Ann Periodontol (2001) 6:99-112. doi:10.1902/annals.2001.6.1.99

17. Kushiyama M, Shimazaki Y, Yamashita Y. Relationship between metabolic syndrome and periodontal disease in Japanese 
adults. J Periodontol (2009) 80:1610-5. doi:10.1902/jop.2009.090218

18. Iwasaki M, Yoshihara A, Miyazaki H. Relationship between screening test for periodontal disease and metabolic syndrome among participants in specific health checkups (in Japanese). J Dent Health (2011) 61(5):573-80.

19. Sheiham A, Watt RG. The common risk factor approach: a rational basis for promoting oral health. Community Dent Oral Epidemiol (2000) 28(6):399-406. doi:10.1034/j.1600-0528. 2000.028006399.x
Conflict of Interest Statement: The authors declare that the research was conducted in the absence of any commercial or financial relationships that could be construed as a potential conflict of interest.

Received: 12 April 2014; paper pending published: 28 April 2014; accepted: 10 May 2014; published online: 28 May 2014.

Citation: Ansai T, Awano S and Soh I (2014) Problems and future approaches for assessment of periodontal disease. Front. Public Health 2:54. doi: 10.3389/fpubh.2014.00054
This article was submitted to Public Health Education and Promotion, a section of the journal Frontiers in Public Health.

Copyright (๑ 2014 Ansai, Awano and Soh. This is an open-access article distributed under the terms of the Creative Commons Attribution License (CC BY). The use, distribution or reproduction in other forums is permitted, provided the original author(s) or licensor are credited and that the original publication in this journal is cited, in accordance with accepted academic practice. No use, distribution or reproduction is permitted which does not comply with these terms. 